\title{
Supporting Information: Complications in the Interpretation of F8T2 Spectra in Terms of Morphology
}

\author{
Liwei Wang and Lewis Rothberg* \\ Department of Chemistry, University of Rochester, Rochester \\ E-mail: lewis.rothberg@rochester.edu
}




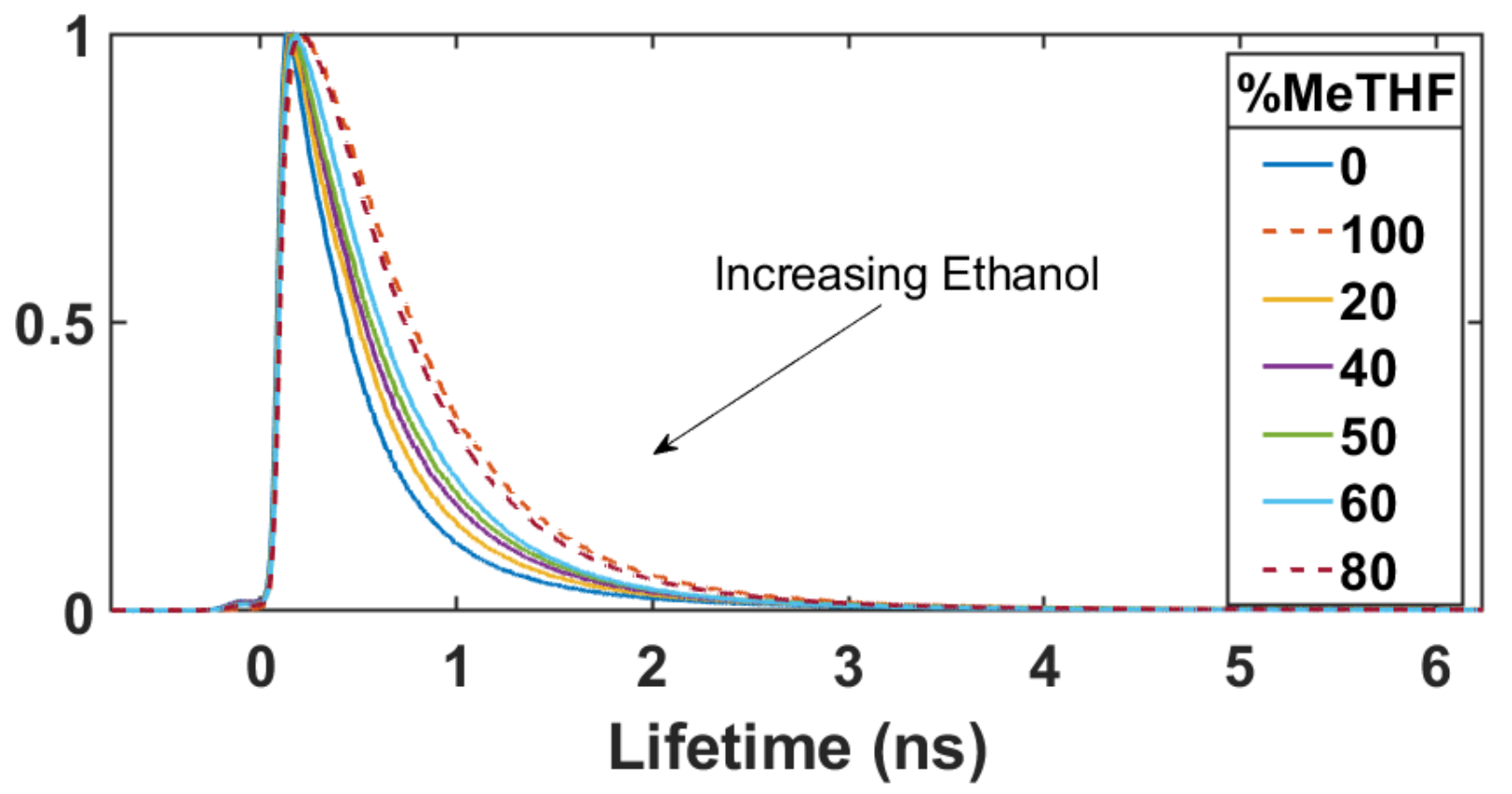

Figure S1: Normalized excited state decay dynamics of F8T2 dissolved in various mixtures of MeTHF (good solvent) and ethanol (poor solvent). The data have not been corrected for the $0.15 \mathrm{~ns}$ duration of the excitation pulse. The orange dotted line represents the dynamics of F8T2 in 100\% MeTHF; red dotted line 80\%; cyan solid line 60\%, green solid line 50\%, purple $40 \%$, yellow $20 \%$, blue $0 \%$

The excited state lifetime of F8T2 decreases with increasing concentration of the poor solvent constituent, reflecting interactions between chromophores induced by aggregation. 

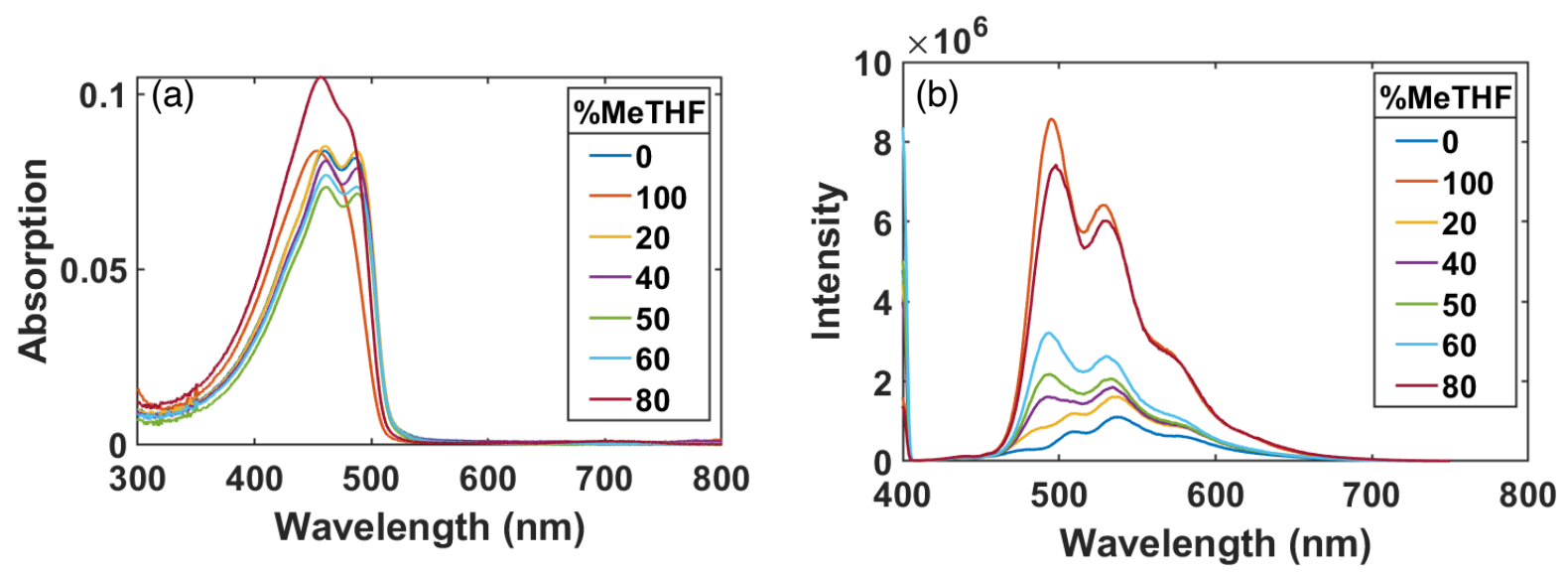

Figure S2: Absorption and photoluminescence spectra of a fixed F8T2 concentration dissolved in mixtures of MeTHF and ethanol. (a) In the absorption, orange represents $100 \%$ MeTHF; red $80 \%$; cyan $60 \%$, green $50 \%$, purple $40 \%$, yellow $20 \%$, blue $0 \%$. (b) In photoluminescence, F8T2 are excited at $400 \mathrm{~nm}$, with same color representation as in absorption.

Both the absorption and photoluminescence spectra fall into two general patterns. As poor solvent content (chromophore aggregation) increases, three phenomena are observed:

1) The absorption spectra develop vibronic structure.

2) The photoluminescence quantum yield decreases.

3) The photoluminescence spectra envelope evolves such that the $0-0$ vibronic band is suppressed relative to the $0-1$ band.

These are all similar to what is observed in polythiophene.
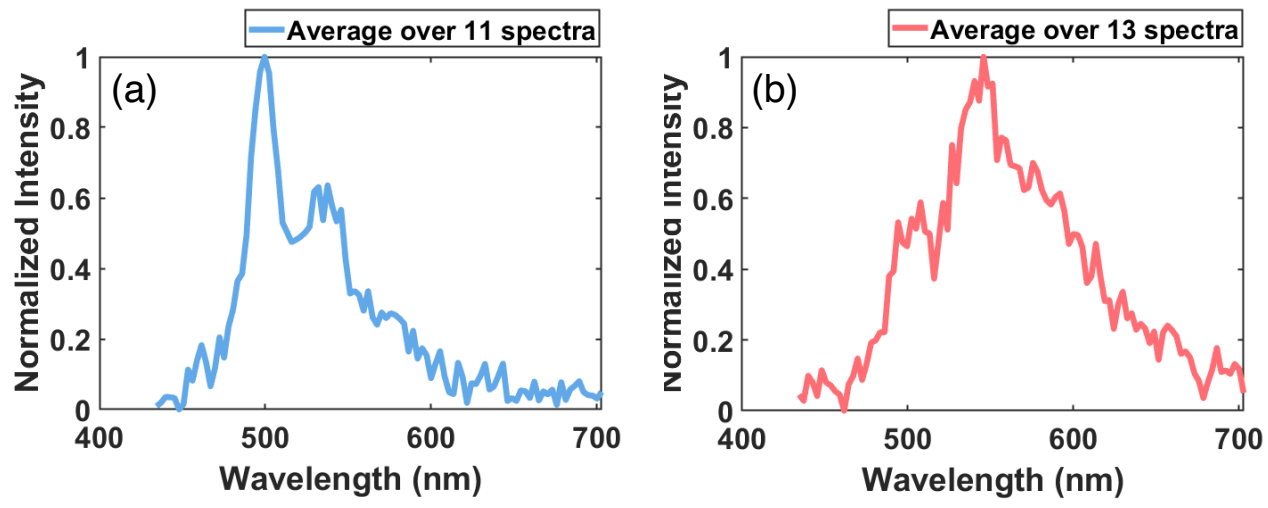

Figure S3: Difference spectra (before drop minus after drop) averaged over many intensity drops analogous to the one displayed in Figure 7 of the text. These are classified into difference spectra with maxima at (a) $500 \mathrm{~nm}$ or (b) between 532 and $543 \mathrm{~nm}$

Note that the difference spectra also exhibit bimodal behavior consistent with the idea that they represent temporary or permanent photobleaching of individual chromophores on a F8T2 chain. 


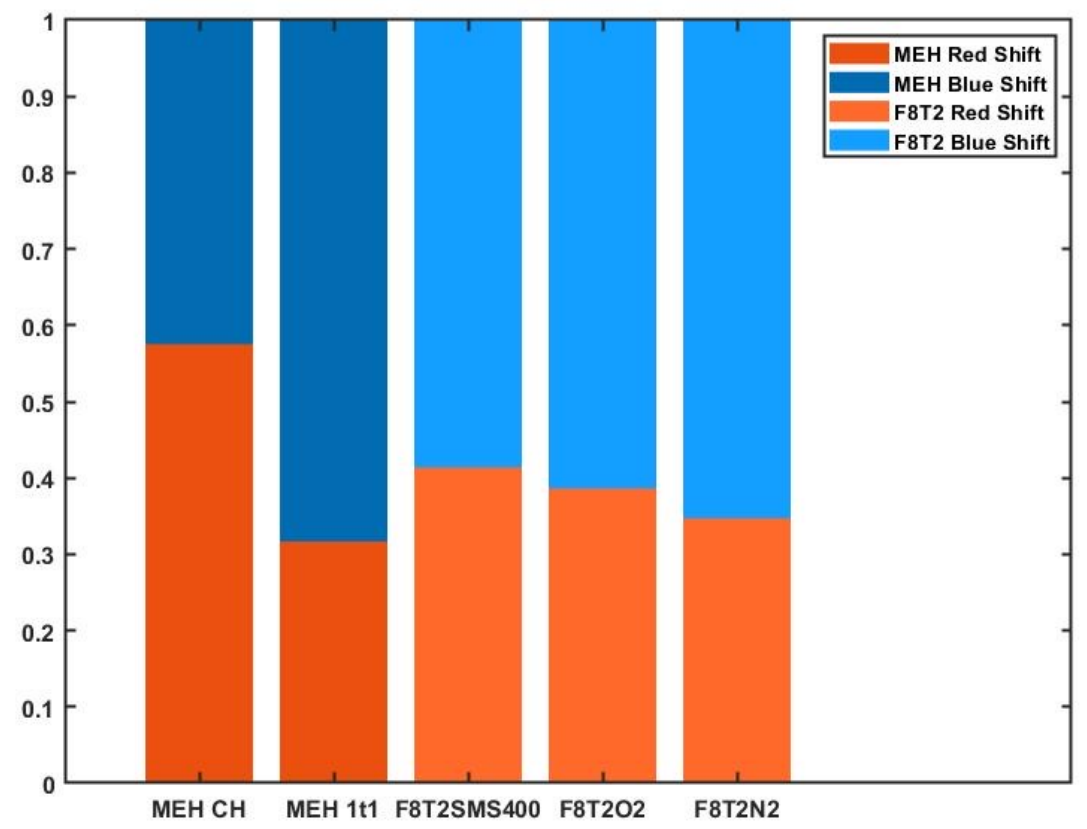

Figure S4: Fraction of chromophores in single chain spectra of conjugated polymers in polystyrene that undergo blue- and red-shifts. Data are for MEH-PPV spun from chloroform, and 1:1 mixture of hexane and chloroform with $488 \mathrm{~nm}$ excitation and $\mathrm{N}_{2}$ protection, F8T2 spun from chloroform with $400 \mathrm{~nm}$ excitation in $\mathrm{N}_{2}$, F8T2 spun from chloroform with $488 \mathrm{~nm}$ excitation in air, and F8T2 spun from chloroform with $488 \mathrm{~nm}$ excitation and $\mathrm{N}_{2}$ protection. 